\title{
Hierarchical cluster analysis in the study of the effect of cytokine expression patterns on endometrial repair and receptivity after hysteroscopic adhesiolysis
}

\author{
Bohan Li, Hua Duan, Sha Wang, Yiyi Wang, Yanan Chang, Zhengchen Guo, Yazhu Li \\ Department of Minimally Invasive Gynecologic Center, Beijing Obstetrics and Gynecology Hospital, Capital Medical University, Beijing, China \\ Contributions: (I) Conception and design: B Li, H Duan; (II) Administrative support: B Li, H Duan; (III) Provision of study materials or patients: \\ S Wang; (IV) Collection and assembly of data: B Li; (V) Data analysis and interpretation: B Li; (VI) Manuscript writing: All authors; (VII) Final \\ approval of manuscript: All authors. \\ Correspondence to: Hua Duan, PhD, MD. Department of Minimally Invasive Gynecologic Center, Beijing Obstetrics and Gynecology Hospital, \\ Capital Medical University, No. 17 Qihelou street, Dongcheng District, Beijing 100006, China. Email: duanhua@ccmu.edu.cn.
}

Background: In a previous study, we reported that amnion promotes endometrial cell growth by regulating cytokines. In this study, hierarchical cluster analysis enabled the evaluation of cytokine expression changes after amnion treatment to be explored by cluster patterns. The role of $I L 1 B$ on endometrial repair and receptivity was revealed.

Methods: A total of 30 patients were recruited in this clinical trial (NCT02496052) of hysteroscopic adhesiolysis. They were randomly allocated into an amnion grafts group (amnion group) and a control group. After hysteroscopic adhesiolysis, a Foley catheter covered with a sterilized freeze-dried amnion graft was inserted into the uterine cavity of the participants in the amnion group, whereas for the control group, a Foley catheter without amnion graft was inserted. After surgery, patient follow-up was done for a year. Uterine exudates were collected every day for seven days after surgery, and analyzed by enzyme-linked immunosorbent assays. Hierarchical cluster analysis was performed to compare expression patterns of each cytokine. Single-gene gene set enrichment analysis and differentially expressed genes enrichment analysis of $I L 1 B$ were performed using NCBI GEO $(\mathrm{N}=151)$ to evaluate its potential mechanisms and impact on endometrial receptivity.

Results: Compared to the control group, cytokine expression patterns of the amnion group revealed significant stratifications, which were highly correlated with the expression levels of $I L 1 B$ on the sixth to seventh day after surgery, improving the pregnant rate. Wilcoxon test revealed significantly low expression levels of $I L 1 B$ in the reduced endometrial receptivity group compared to the normal group. Moreover, gene set enrichment analysis showed that lysosomes, cell cycle, and calcium signaling pathways were associated with the biological processes in which $I L 1 B$ plays a role. Screening and enrichment analyses of differentially expressed genes further verified the mechanisms of action of $I L 1 B$ on endometrial repair and receptivity recovery.

Conclusions: Amnion promotes endometrial repair and receptivity by altering the expression levels and patterns of $I L 1 B$. Furthermore, by affecting lysosomal, cell cycle, and calcium signaling pathways, IL1B may be one of the factors involved in endometrial repair and receptivity recovery.

Keywords: Intrauterine adhesion; cytokines; hierarchical cluster analysis model; endometrial repair; endometrial receptivity

Submitted Jan 14, 2021. Accepted for publication Mar 14, 2021.

doi: $10.21037 /$ atm-21-195

View this article at: http://dx.doi.org/10.21037/atm-21-195 


\section{Introduction}

Intrauterine adhesions (IUAs), also known as the Asherman syndrome, are caused by damage to the basal layer of the endometrium and can partially or completely obliterate the uterine cavity (1). Clinical conditions associated with IUAs include menstrual disorders (oligomenorrhea or secondary amenorrhea), infertility, recurrent spontaneous abortion, and premature labor. Infertility is one of the main reasons as to why IUA patients seek clinical treatment (2). However, it is challenging to treat infertility. Currently, the most common therapeutic option for IUA is hysteroscopic adhesiolysis. Intrauterine devices (IUD) and Foley catheters are used to prevent adhesion reformation after surgery, and the rate of adhesion reformation ranges between $30 \%$ $66 \%$ even after the use of IUD and Foley catheters (3). Furthermore, postoperative pregnancy rate has been reported to be about $40 \%$ (4). Even though re-adhesion of the uterine cavity after hysteroscopic adhesiolysis is affected by many factors, inflammation is one of the main causes of scar formation. Previous studies on inflammatory responses focused on the expression of immune-related proteins or cytokines (5-7). However, postoperative inflammation is a dynamic process. The early stages of traumatic inflammation and repair include two phases, the first being inflammatory exudation and neovascularization (inflammatory phase), and the second being fibrosis as well as tissue proliferation and migration (repair phase) (8). Orhue et al. (9) reported that wound healing begins approximately seven days after hysteroscopic adhesiolysis, while Xin et al. (7) showed that the two aforementioned phases have a high correlation with inflammatory responses, which are dominated by macrophage transformations. Immune responses are significantly different in the inflammatory phase compared to the repair phase.

In our previous study, we reported that human amniotic mesenchymal stromal cells promote endometrial cell proliferation by regulating cytokines (10). Amnion gifts exhibit a large number of cytokines and receptors that can enhance injury repair. It accelerates tissue repair by inhibiting the expression levels of TNF- $\alpha$ and VEGF in the wounded area and prevents epithelial cell apoptosis by stimulating cell migration and adhesion. Moreover, in a previous clinical study, we showed that sterilized freezedried amnion grafts promote endometrial repair and receptivity after surgery (1). These findings show that amnion graft activity is exerted through cytokine regulation. Differences in expression patterns and cytokine types in different periods after surgery play an important role in endometrial receptivity recovery.

Hierarchical cluster analysis (HCA), also referred to as hierarchical or tree clustering, is a cluster analysis method that is commonly used in data search or mining to establish a hierarchical structure of clustering (11). This method uses a randomized clustering algorithm to group factors with similar characteristics into clusters. Based on the available features, clustering analysis classifies mixed factors into homogenous groups. Each cluster has its own signature for identification. This approach has been applied in many clinical fields $(12,13)$. In this study, HCA, in combination with regression analysis effectively clustered the stages of inflammation and cytokines. The clusters enabled the evaluation of differences in cytokine expression levels and patterns between the amnion group and the control group.

Few studies have evaluated the effects of changing cytokine expression patterns in different inflammation stages on the endometrium, after surgery. Therefore, we evaluated the differences in cytokine expression patterns at various stages of post-operative inflammation. Even though current public databases lack the genome-wide expression data for intrauterine adhesions, data on infertility associated endometrial damage is widely available. We analyzed these data to determine the biological mechanisms of cytokines in the process of endometrial repair and receptivity recovery. We present the following article in accordance with the MDAR reporting checklist (available at http://dx.doi. org/10.21037/atm-21-195).

\section{Methods}

\section{Study design}

This was a randomized controlled clinical trial (Trial Registration Number NCT02496052). A total of 30 patients were recruited in this study and randomly allocated into the amnion and control groups with 15 cases each. Randomization was done using a computer algorithm. Hysteroscopic adhesiolysis was performed on participants using intrauterine adhesions, and Foley catheters with or without freeze-dried amnion grafts were placed on the participants after surgery. Patient follow up was done at 3 months, 6 months, and 1 year after surgery. Endometrial thickness was assessed by ultrasound examination while pregnancy status was recorded at each follow-up visit. During follow-up, hysteroscopy was performed to assess the American Fertility Society (AFS) again at 3 months 

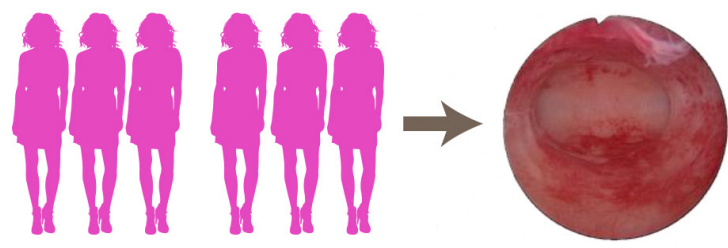

Amnionn vs. control

15 vs. 15

Hysteroscopic adhesiolysis

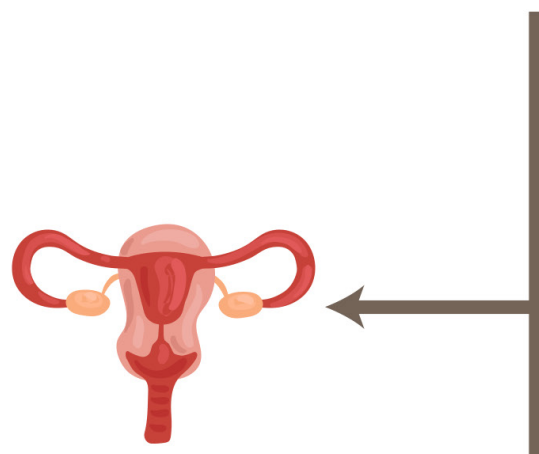

Endometrial thickness changes of each group

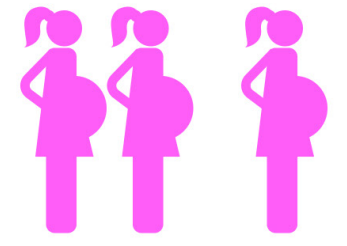

Pregnancy as endpiont pregnancy rate of each group
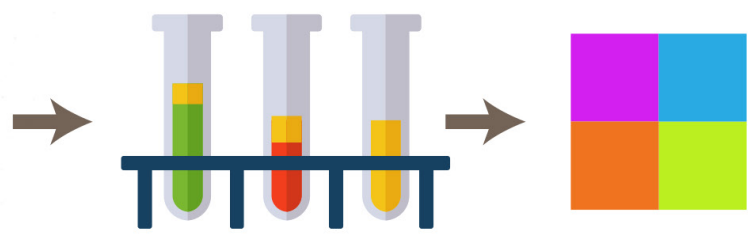

ELISA of cytokines for 7 days after surgery

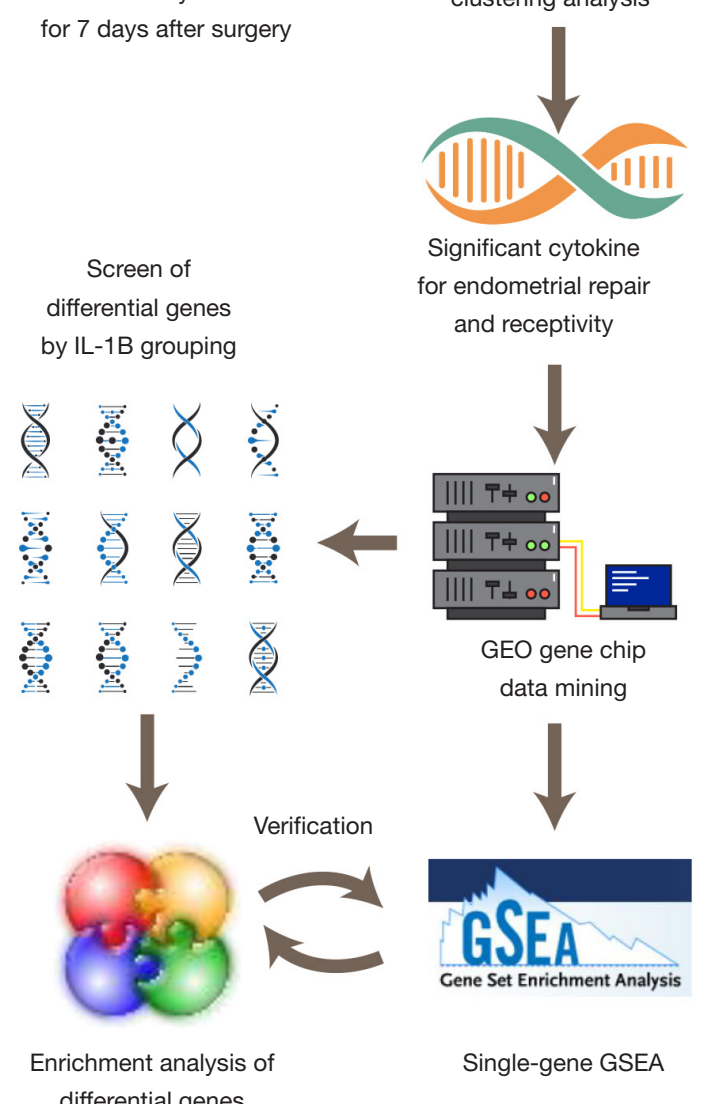

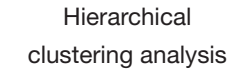

Significant cytokine

or endometrial repai

GEO gene chip

Figure 1 Flow diagram of the study design.

postoperatively to observe their endometrial recovery. The median follow-up time was 7.5 months. Uterine exudates were collected at $3 \mathrm{~h}, 1 \mathrm{~d}, 2 \mathrm{~d}, 3 \mathrm{~d}, 4 \mathrm{~d}, 5 \mathrm{~d}, 6 \mathrm{~d}$ and $7 \mathrm{~d}$ after surgery. Then, the enzyme-linked immunosorbent assay kit was used to determine the levels of $I L 1 B$ [interleukin (IL)-1 $\beta$ ], tumor necrosis factor (TNF)- $\alpha$, and vascular endothelial growth factor (VEGF) cytokines in the exudate. Furthermore, postoperative cytokine concentrations and patterns in the two groups of patients were compared using hierarchical cluster analysis to determine the effects of cytokines on endometrial receptivity recovery.
Furthermore, we used the GEO database to establish the effects of cytokines on endometrial receptivity, as well as the biological mechanisms involved (Figure 1).

\section{Study participants}

Thirty patients with severe IUA who had been admitted to the Beijing Obstetrics and Gynecology Hospital affiliated to Capital Medical University between February 2017 and December 2018 were recruited in this study. Using a computer algorithm, patients were randomly assigned 


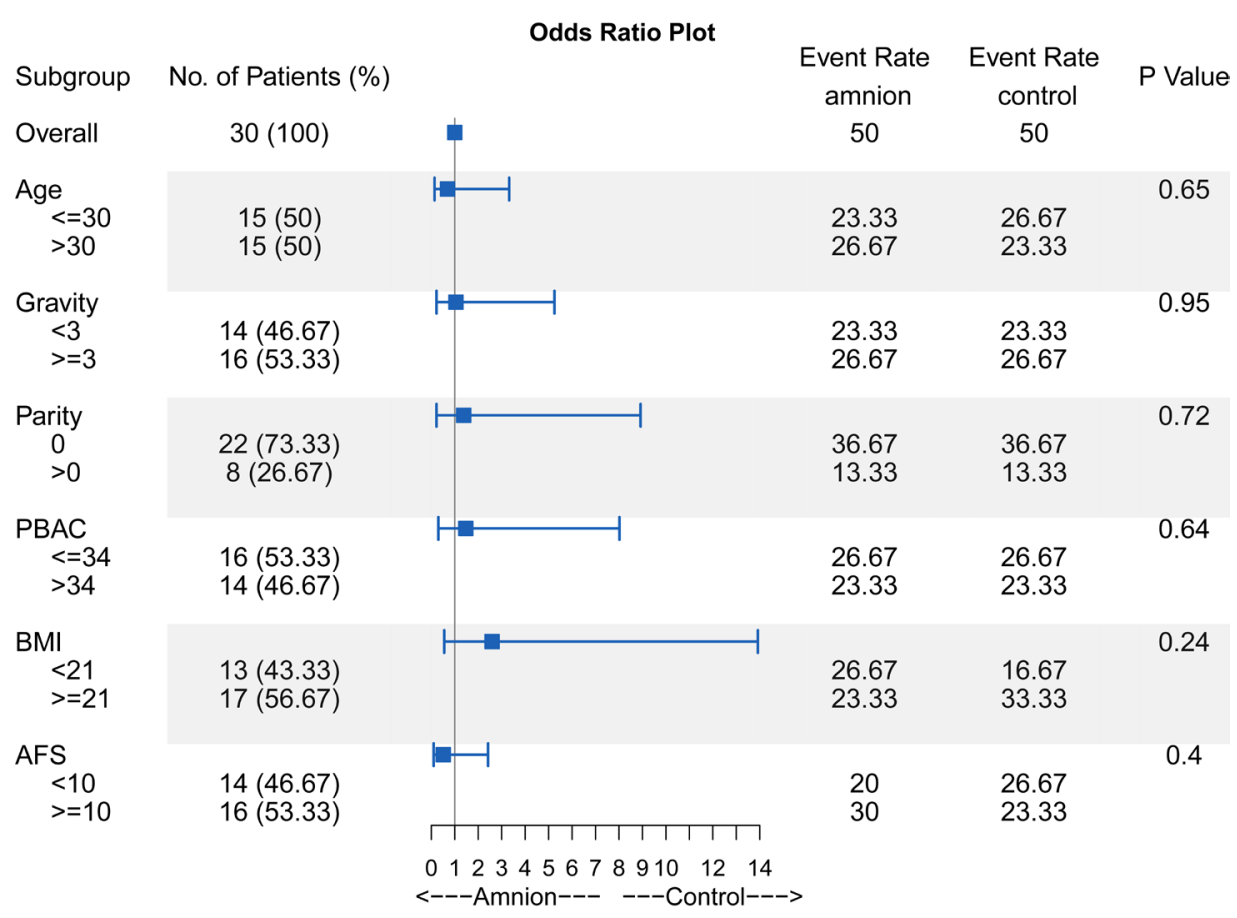

Figure 2 Clinical characteristics of study participants. All the clinical characteristics above were not statistically different between the two groups $(\mathrm{P}>0.05)$. PBAC, pictorial blood loss assessment chart; BMI, Body Mass Index; AFS, American Fertility Society.

to the amnion group and the control group with 15 cases each. Ethical approval for this study was obtained from the Research Ethics Committee of the Beijing Obstetrics and Gynecology Hospital. Experiments were performed (under protocol number 2017-KY-082-02) according to the Helsinki Declaration of 1975 (revised in 2013). Patients eligible for surgery were required to sign an informed consent the night before surgery. The AFS scoring system was used to evaluate intrauterine adhesions. The inclusion criteria were: (I) clinically diagnosed intrauterine adhesions; (II) adhesion score $\geq 9$ points; (III) normal ovarian function tests; (IV) aged between 18-40 years; and (V) normal results from Thinprep cytologic test. Exclusion criteria were: (I) patients with adenomyosis complications; (II) presence of uterine fibroids; (III) patients with developmental abnormalities of the uterus; (IV) those with other diseases that cause infertility or hypomenorrhea; (V) those who had been treated with hormones within 3 months before surgery; (VI) those with endocrine diseases, such as hyperthyroidism and adrenal hyperfunction; and (VII) patients who were not able to undergo the operation (1). The mean ages of patients in the amnion and control groups were 30.2 and 29.7, respectively. Statistical analyses of the basic demographic properties before grouping were performed by generalized linear regression, and results were corrected for individual characteristics based on the incorporated factors (14) (Figure 2).

\section{Sterilized freeze-dried amnion grafts}

Jiangxi Rui Ji Biotechnology (Jiangxi, China) provided the sterilized freeze-dried amnion grafts used in this study, and its production processes were as follows. Fresh amniotic tissues were collected and sterilized by low-temperature freeze-drying as well as cobalt-60 radiation. The above steps allow the amniotic cells to remain in a "dormant" state, thereby guaranteeing their ability to be stored and transported at room temperature in a sealed package.

\section{Process flow}

A size-20 Foley catheter was routinely used after surgery for patients in the control group. For the amnion group, the balloon portion of the Foley catheter was covered with sterilized freeze-dried amnion grafts and hydrated in sterile normal saline for 10 minutes before insertion. The size of each amnion graft was $30 \mathrm{~mm} \times 20 \mathrm{~mm}$. Two amnion grafts were applied to each Foley catheter with the epithelial 
amnion membrane surface facing outward. In contrast, for the control group, the Foley catheter was not covered with the gift. Then, under ultrasonographic guidance, the Foley catheter was inserted into the uterine cavity. After insertion, the balloon was inflated for 2-3 minutes with $8-10 \mathrm{~mL}$ normal saline to ensure that the amnion graft was fully adhered to the uterine cavity. After inflation, about $3-5 \mathrm{~mL}$ of the normal saline solution was drawn from the balloon leaving an average of $5 \mathrm{~mL}$ normal saline (1). The Foley catheter remained in place for a week, with the uterine exudate being collected every day. After day 7, the balloon was deflated, and the catheter removed through an outpatient surgical procedure.

\section{Hysteroscopic adbesiolysis}

Hysteroscopic adhesiolysis was performed by an experienced hysteroscopist (H Duan) in all patients under general anaesthesia. Two misoprostol tablets (200 $\mu \mathrm{g}$ each), were vaginally administered for cervical priming the night before the procedure. A bipolar resectoscope with an $8-\mathrm{mm}$ sheath and a $4-\mathrm{mm} 12^{\circ}$ telescope (Olympus Optical Company, Tokyo, Japan) was used after cervical dilation with a Hegar size 10 cervical dilator. Ultrasonography was routinely performed during the procedures. Laparoscopy was used to inspect the pelvic pathologies, such as endometriosis, and to check tubal patency at the end of the hysteroscopic surgery. Normal saline, delivered by an automatic hysteroscopic dilatation pump at a rate of $280-300 \mathrm{~mL} / \mathrm{min}$ under an intrauterine pressure of $100 \mathrm{mmHg}$, was used as the dilatation medium.

After assessing the location, extent, and severity of the IUAs, they were resected by a needle or loop diathermy, using an electrosurgical generator voltage set at $320 \mathrm{~W}$ for the cutting mode. Then, fluid volume was recorded by a modified automated fluid management system. The operating surgeon determined when complete adhesiolysis had been achieved for all participants, and this was verified by the normal panoramic view of the uterine cavity under direct hysteroscopic visualization. Adhesiolysis was evidenced by an adequate uterine cavity, no signs of IUA, and visible bilateral uterine horn with or without fallopian ostium.

\section{Enzyme-linked immunosorbent assay}

Concentrations of $I L 1 B, T N F-\alpha$ and VEGF in the uterine exudate collected at different time points after hysteroscopic adhesiolysis were determined using a human $I L 1 B$, TNF- $\alpha$ and VEGF enzyme-linked immunosorbent assay (ELISA) kit (Uscn Life Science Inc, Wuhan, China) according to the manufacturer's instructions. Samples or standard $(50 \mu \mathrm{L})$ were separately added to each well followed by the addition of the detection reagent $(50 \mu \mathrm{L})$. Then, the mixture was incubated for $30 \mathrm{~min}$ at $37^{\circ} \mathrm{C}$. Subsequently, $50 \mu \mathrm{L}$ of color development reagent $A$ and $50 \mu \mathrm{L}$ of reagent $B$ were added and incubation was done for $15 \mathrm{~min}$ at $37^{\circ} \mathrm{C}$. Furthermore, $50 \mu \mathrm{L}$ of the stop solution was added to each well, and optical density (OD) was read at $450 \mathrm{~nm}$. Washing of the plate during these procedures were done according to the routine ELISA method. Linear regression equation of a standard curve was calculated using the concentration and OD value of the standard substance according to a procedure described by Yolken RH. OD values of the samples were substituted into the equation to calculate sample concentrations in $\mathrm{ng} / \mathrm{L}$. Then, the concentration was multiplied by a dilution factor to obtain the actual cytokine concentration.

\section{Hierarchical clustering analysis}

Regression analysis was used to compare the expression levels of different cytokines. Expression levels of cytokines were first normalized to balance the influence of weights on the trend. The Min-max normalization approach was performed to have the data interval between 0 and 1 for various cytokines. The specific formula used was: $x^{\prime}=\frac{x-x_{\min }}{x_{\max }}$. Furthermore, hierarchical cluster analysis was performed on the regression coefficients ( $\beta$ value) of different cytokines based on the changing trend and time points. Distance between the two coefficients was calculated using the formula Dist $=\left\|X_{i}-X_{j}\right\|_{2}$. The height between two clusters was expressed as Height $=\operatorname{Max}\{d(a, b), a \in A, b \in B\}$. Different stages of inflammation were determined through cluster analysis (11).

\section{Source of the microarray data}

In this study, mRNA microarray datasets were retrieved from the GEO database under the accession numbers GSE58144, GSE71835, GSE92324 and GSE103465 $(15,16)$. The inclusion criteria were: (I) age ranging between 20 and 40 years; (II) history of uterine cavity procedure; (III) normal levels of serum follicle stimulating hormone (FSH), luteinizing hormone (LH), estradiol, progesterone, anti-Mullerian hormone (AMH) and prolactin. Patients with polycystic ovarian disease, endometriosis, hormonal 
Table 1 Overview of details for original studies included in a integration analysis of GWES for endometriosis

\begin{tabular}{llccc}
\hline Record & Region & Platform & Gene symbols & Simple (infertility vs. normal) \\
\hline GSE92324 & endometrium & GPL10558 & 34694 & 10 vs. 8 \\
GSE58144 & endometrium & GPL15789 & 34127 & 43 vs. 72 \\
GSE103465 & endometrium & GPL16043 & 12284 & 3 vs. 3 \\
GSE71835 & endometrium & GPL10558 & 34694 & 6 vs. 6 \\
\hline
\end{tabular}

abnormalities, autoimmune disorders were excluded from the study. Detailed information of the samples in the data sets are shown in Table 1.

\section{Data processing and integration}

Microarray datasets pre-processing and normalization were performed using the affy package in $\mathrm{R}$ software, based on the raw data of the Affymetrix platform (17). The following methods were used: robust multi-array average (RMA, for background correction) (18), quantile (for normalization) (19), pmonly method (perfect match correction) (20) and the median polish (as a summary method) (21). Normalized data for microarray datasets based on other platforms (including Illumina (2 dataset) and Agilent (1 datasets)), were downloaded and used in the subsequent analyses. Data that were not processed using the RMA method were converted to $\log$ format (base 2). Annotation files for different microarray platforms were retrieved from the NCBI GEO database (22). Surrogate variable analysis (SVA, in the $\mathrm{R}$ environment, version 3.6.2) (23) was used for integration analysis and correction of batch-to-batch differences, following the published recommendations for this type of study (21). Prior to SVA analysis, all data were transformed to the $\log 2$ format. During the data integration process, the number of genes covered by GWES differed for the different batches. Principal component analysis (PCA) was used to verify the integrated data without exerting a significant impact on data analysis due to batch effect of different platforms and to ensure that data reflected the biological characteristics of endometriosis.

\section{Enrichment analysis}

Gene-set enrichment analysis (GSEA) was performed using the "ClusterProfiler" (24) package in R. The Broad Molecular Signatures Database (MSigDB v7.0) dataset in the Kyoto Encyclopedia of Genes and Genome (KEGG) (c2.cp.kegg.v7.0.symbols) was used. This database summarizes and presents specific well-defined biological states and pathway processes. The GSEA program was run with 1,000 permutations for statistical significance estimation, and correlations between $I L 1 B$ and other genes was used to rank all genes.

ClueGo (based on Cytoscape, version 3.7.2) was performed for functional category enrichment analysis of differentially expressed genes (DEGs) to verify GSEA results. GOplot package in $\mathrm{R}$ software was used to visualize enrichment analysis results and their regulatory conditions. The formula used for calculating the $\mathrm{Z}$-score was zscore $=\frac{\text { (up-down })}{\sqrt{\text { count }}}$. The Z-score indicates whether the GO term is likely to be decreased (negative value) or increased (positive value).

\section{Statistical analyses}

The statistical test used for the comparison of $I L 1 B$ levels between the normal group and the reduced endometrial receptivity group is the $t$-test or paired $t$ test. Prognostic curves were generated through the Kaplan-Meier method while log-rank tests were utilized to identify significant differences in pregnancy outcomes. Fisher's exact test was used for enrichment analysis of DEGs. Pearson's correlation coefficient analysis was performed for the single gene GSEA of IL1B. The "Limma" package (25) in R software was used for DEGs analysis of $I L 1 B$-high and IL1B-low samples. Multiple testing adjustments (False Discovery Rate, FDR $<0.05$ ) and $\log _{2}$ ratio of gene expression fold change ( $\log _{2}$ Fold Change $>1$ ) was used to determine statistical significance. Statistical analyses were performed using GraphPad Prism software (GraphPad Software, San Diego, California) or R software.

\section{Results}

\section{Cytokine expression patterns and clinical outcomes}

Regression coefficients of cytokine levels at various 
time points from the first day to the seventh day after hysteroscopic adhesiolysis were determined to establish the changing trends at each time point. Further, hierarchical cluster analysis was performed to group cytokines and time points (Figure 3A). Cytokine grouping showed that TNF- $\alpha$ and VEGF for the amnion group and the control group were classified into one cluster. These findings indicate that the expression patterns of TNF- $\alpha$ and VEGF cytokines in the two groups were similar. On the contrary, the distance (height) between $I L 1 B$ and the other two cytokines was significantly larger in the amnion group compared to the distance in the control group (height study $=1.67$ and height control $=1.26$, respectively). Cluster analysis for time points showed that the amnion group had distinct time stages compared to the control group. Based on the $6^{\text {th }}$ day after surgery (height $=1.40$ ), the amnion group was divided into two phases. Comparisons of cytokine expression levels before and after the 6-day post-operation period showed that the amnion group exhibited significantly different expression levels of $I L 1 B$ when compared to the control group. In the amnion group, $I L 1 B$ increased significantly between day 6 and day 7 after hysteroscopic adhesiolysis, consistent with timing of the repair process in the endometrium after hysteroscopic adhesiolysis (9). Cytokine concentrations at each time point are shown in Tables S1,S2,S3.

Follow-up results were also analyzed. Postoperatively, endometrial thickness was found to be significantly increased in both groups compared to preoperative thickness $(\mathrm{P}<0.05)$. Mean values of changes in endometrial thickness were higher in the amnion group than in the control group, however, there was no statistical difference $(\mathrm{P}>0.05)$ (Figure 3B). The decrease in postoperative AFS levels between the two groups was significant $(\mathrm{P}=0.003)$, implying better endometrial recovery in the amnion group, as shown in Figure 3C.

During the follow-up period, a total of seven women in the amnion group achieved pregnancy. Of these, five pregnancies occurred naturally, while two occurred after in vitro fertilization and embryonic transfer. Spontaneous abortion during the first trimester was reported in one of the seven pregnancies of the amnion group. The remaining six pregnancies were viable at the time of the last follow-up. In the control group, three pregnancies were reported, and all had occurred after in vitro fertilization and embryonic transfer. Among these pregnancies, no spontaneous abortion was reported during the first trimester, and all three pregnancies were viable at the last follow-up. Logrank analysis revealed that there were statistical differences between the two groups $(\mathrm{P}=0.0097)$ (Figure 3D). In combination with hierarchical cluster analysis, we inferred that $I L 1 B$ may play a role in the process of receptivity recovery and repair of the endometrium after hysteroscopic adhesiolysis.

\section{Effect of IL1B on repair and endometrial receptivity}

Further, we evaluated the effect of $I L 1 B$ on receptivity recovery and repair of endometrium. GWES datasets of premenstrual endometrial tissue specimens from patients with abnormal endometrial receptivity and history of uterine cavity procedure were retrieved from the GEO database for analysis. PCA of the different datasets was performed, after which batch differences between the datasets were integrated and analyzed. The combined data showed no significant batch effects (Figure 4A). In addition, the two groups could be distinguished after dimensionality reduction by PCA (Figure S1). Further, the expression levels of $I L 1 B$ between the normal group and the reduced endometrial receptivity group were compared. The expression levels of $I L 1 B$ for patients in the reduced endometrial receptivity group was significantly higher compared to $I L 1 B$ expression levels for patients in the normal group $(\mathrm{P}<0.05)$ (Figure $4 B)$.

\section{KEGG enrichment analysis of ILIB}

Gene Set Enrichment Analysis (GSEA) was used to test for single gene enrichment of pathways associated with $I L 1 B$. To comprehensively elucidate the biological pathways associated with $I L 1 B$ in endometrial recovery, all integrated genes were included for enrichment analysis. Pearson correlation analysis was used to rank all genes according to their association with $I L 1 B$. The probable biological pathways associated with $I L 1 B$ functions were analysed based on gene ranking to identify the pathways enriched by these genes. The expression levels of $I L 1 B$ and associated genes were significantly functionally enriched in lysosomal, cell cycle and calcium signalling pathways, with normalized enrichment scores of $0.62,0.51$, and 0.49 , respectively (Figure 5A). The FDR scores for lysosomal, cell cycle and calcium signalling pathways were $0.006,0.006$ and 0.0006 , respectively (Figure $5 B$ ). These findings indicate that the mechanisms by which $I L 1 B$ promotes receptivity recovery and repair of the endometrium is associated with lysosomal, cell cycle and calcium signalling pathways.

The correlations between genes in the gene-sets 
A
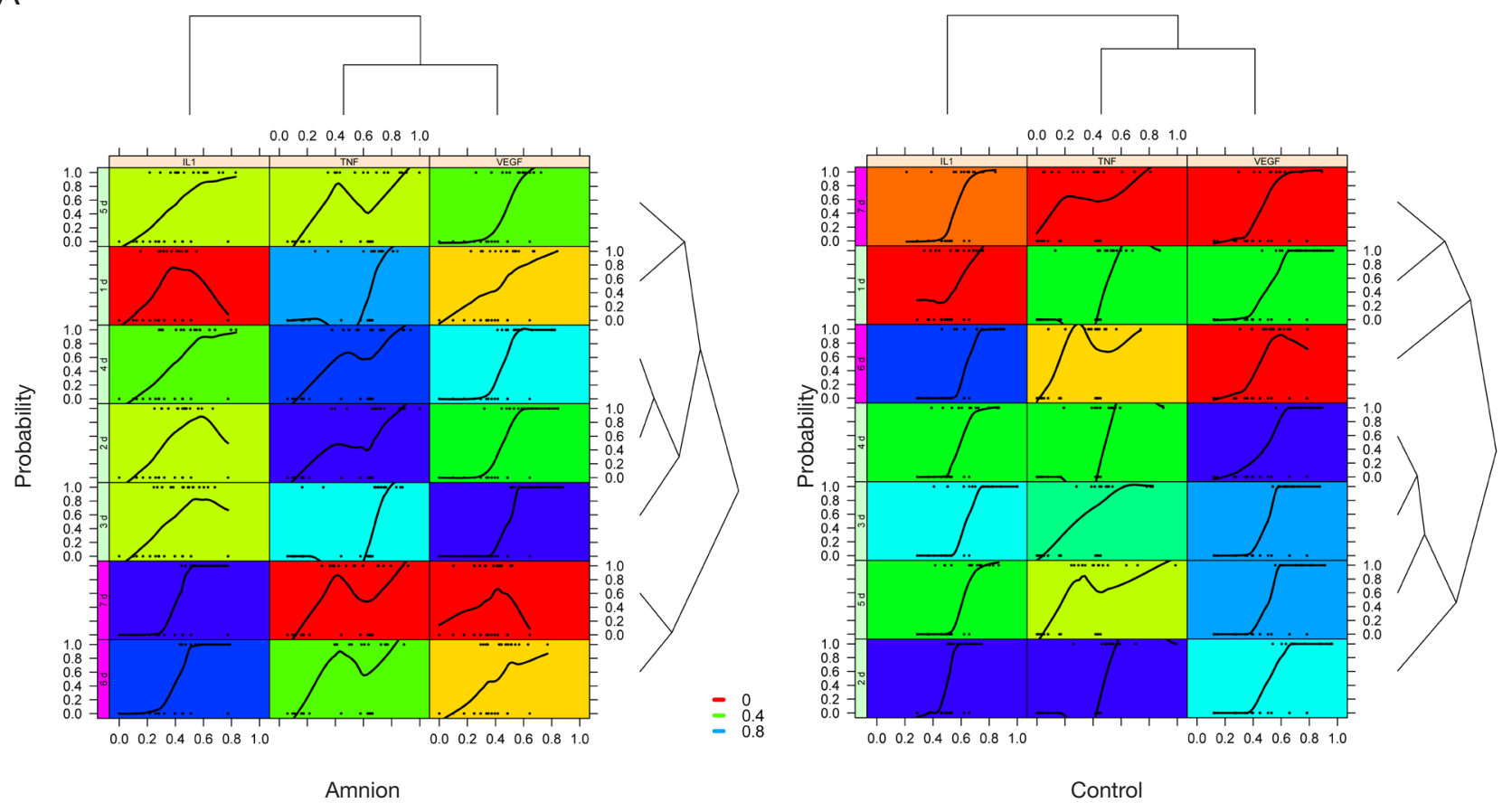

B

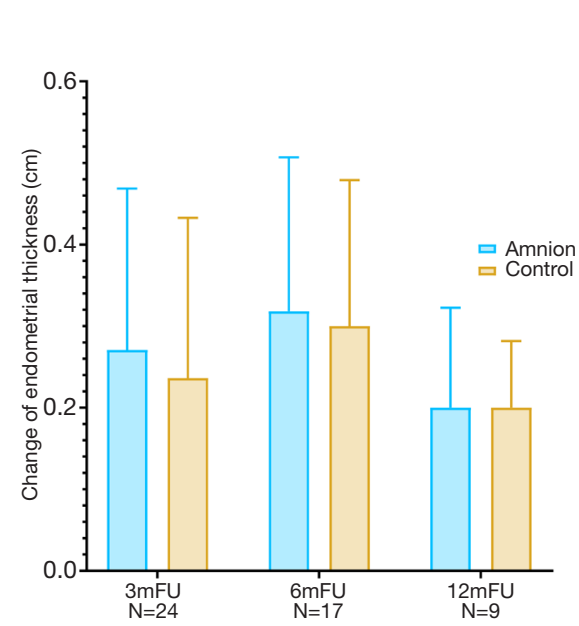

C

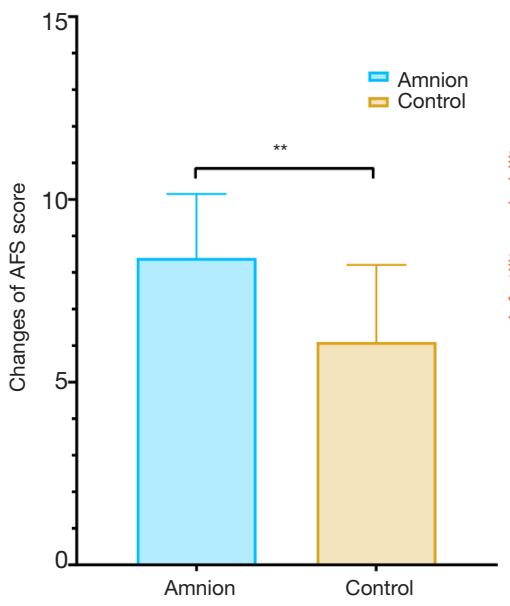

$\mathrm{D}$

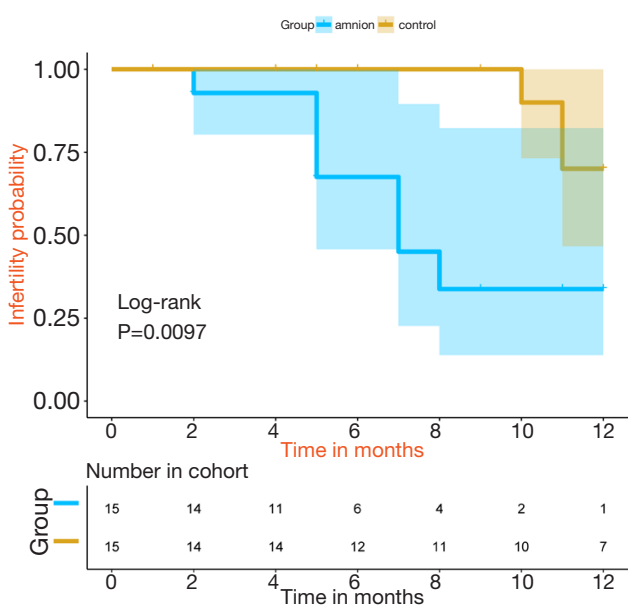

Figure 3 Cytokine expression patterns and clinical outcomes. (A) Hierarchical cluster analysis of cytokine expression patterns. Background colors of each panel map to normalized coefficient values, and the link between colors and values. Blue represents rapid growth, while red is slow. The upper part of the heat map represents cluster dendrograms of cytokines, while the right side is time points with red representing the 6-7th day after surgery. (B) Histograms of changes in endometrial thickness after surgery. Blue bars represent mean changes in the amnion group, while orange bars represent the control group. FU, follow-up; m, months. (C) Histograms of changes in AFS scores after surgery. Blue bars represent mean changes in the amnion group, while orange bars represent the control group. ${ }^{* *}, \mathrm{P}<0.01$. (D) KaplanMeier curves of postoperative pregnant status analysis for amnion and control groups. Log-rank $\mathrm{P}=0.0097$ showed a significant difference between the two groups. Number in cohort table reveals the number of people at each time point who have not yet achieved the endpoint and are still in the follow-up cohort. 
A

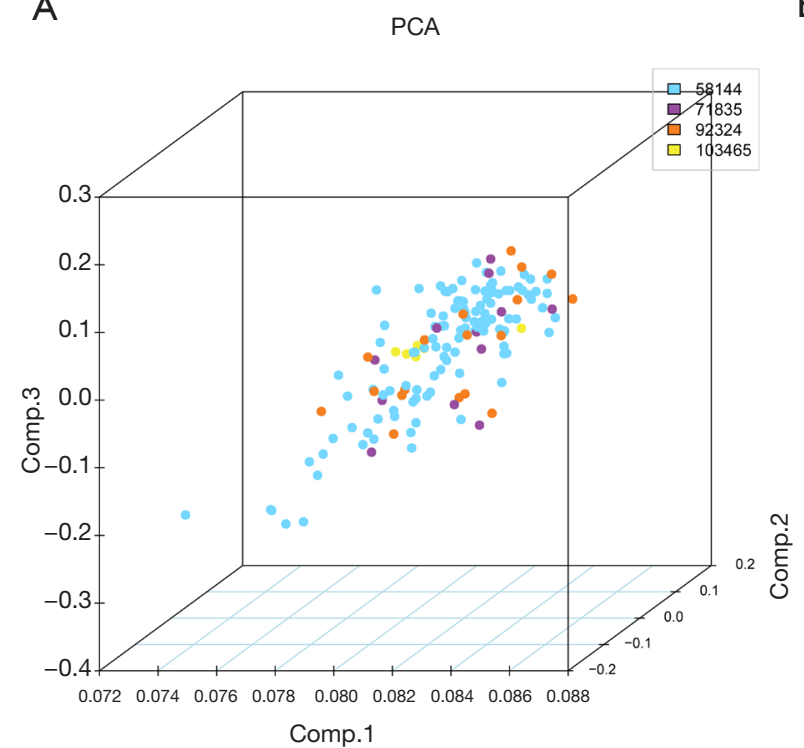

B

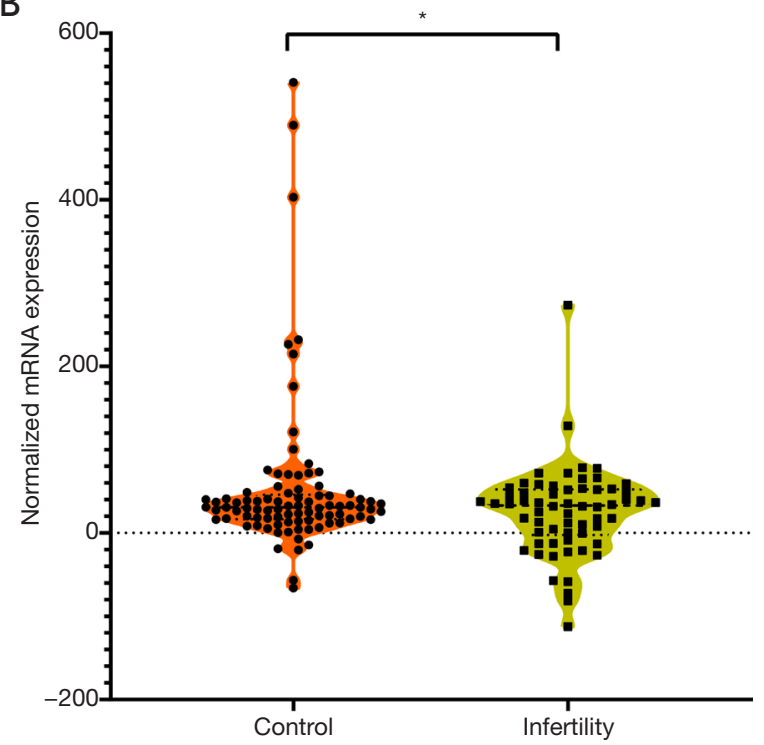

Figure 4 Analysis of $I L 1 B$ expression levels in the integrated data set. (A) PCA plot of the data for the different datasets. Distribution of the two groups can be seen in Figure S1. (B) Violin plot of $I L 1 B$ expression. *, $\mathrm{P}<0.05$.

from KEGG pathway and $I L 1 B$ were further studied by performing a hierarchical cluster analysis of the genes in the lysosome, cell cycle and calcium signalling pathways according to the expression levels of $I L 1 B$. The expression levels of genes in these pathways were shown to increase with an increase in $I L 1 B$ levels with similar expression patterns (Figure 6). In addition, the expression levels of $C T S A$, cyclin-dependent kinases $(C D K s)$ and $C D 38$ genes in the lysosome, cell cycle and calcium signalling pathways, respectively, were highly correlated with $I L 1 B$ expression levels.

\section{Biological functions associated with IL1B over-expression}

To verify the results from Gene Set Enrichment Analysis and to evaluate the biological functions associated with $I L 1 B$ over-expression, we grouped patients into $I L 1 B$ high $(\mathrm{n}=50)$ and $I L 1 B$-low $(\mathrm{n}=50)$ groups. Then, DEGs analysis of the two groups was performed. Selected DEGs were subjected to GO and KEGG enrichment analyses. ClueGO enrichment analysis revealed that the enrichment results were consistent with GSEA results. The gene expression fold change results revealed that lysosomal, cell cycle and calcium signaling pathways are positively enriched in samples with elevated expression levels of $I L 1 B$ compared to samples with suppressed expression levels of IL1B (Figure $7 A$ ).
In addition, we calculated the specific GO enrichment of DEGs. Function and location network diagram of DEGs enrichment is shown in Figure 7B. In the biological processes (BP), the mainly enriched terms were "cell division" and "cell cycle" (FDR $=5.05 \times 10^{-22}$ and $2.46 \times 10^{-20}$, respectively). These terms are associated with the cell cycle pathway. Cellular components (CC) that were enriched were cyclin-dependent protein serine/threonine kinase regulator activity (FDR $=2.32 \times 10^{-14}$ ) and cation-transporting ATPase activity $\left(\mathrm{FDR}=4.08 \times 10^{-13}\right)$ terms, which are implicated in the cell cycle pathway. Molecular function (MF) terms that were enriched were the "vacuole" and "lysosome" (FDR $=2.55 \times 10^{-22}$ and $7.38 \times 10^{-21}$, respectively), which are associated with the lysosomal pathway. The enriched genes in each GO terms are shown in Figure 7C.

\section{Discussion}

Hysteroscopic lysis of adhesions is an effective surgical treatment approach for uterine scars. However, there are no effective therapeutic approaches that can prevent recurrence (26). Hysteroscopic surgery is the gold standard for the management of severe IUAs. This technique is limited by the application of energy devices, such as electrodes, which produce electrocaloric effects on normal endometrial and myometrium tissues while separating and removing scar tissues in the uterine cavity. These effects 
A
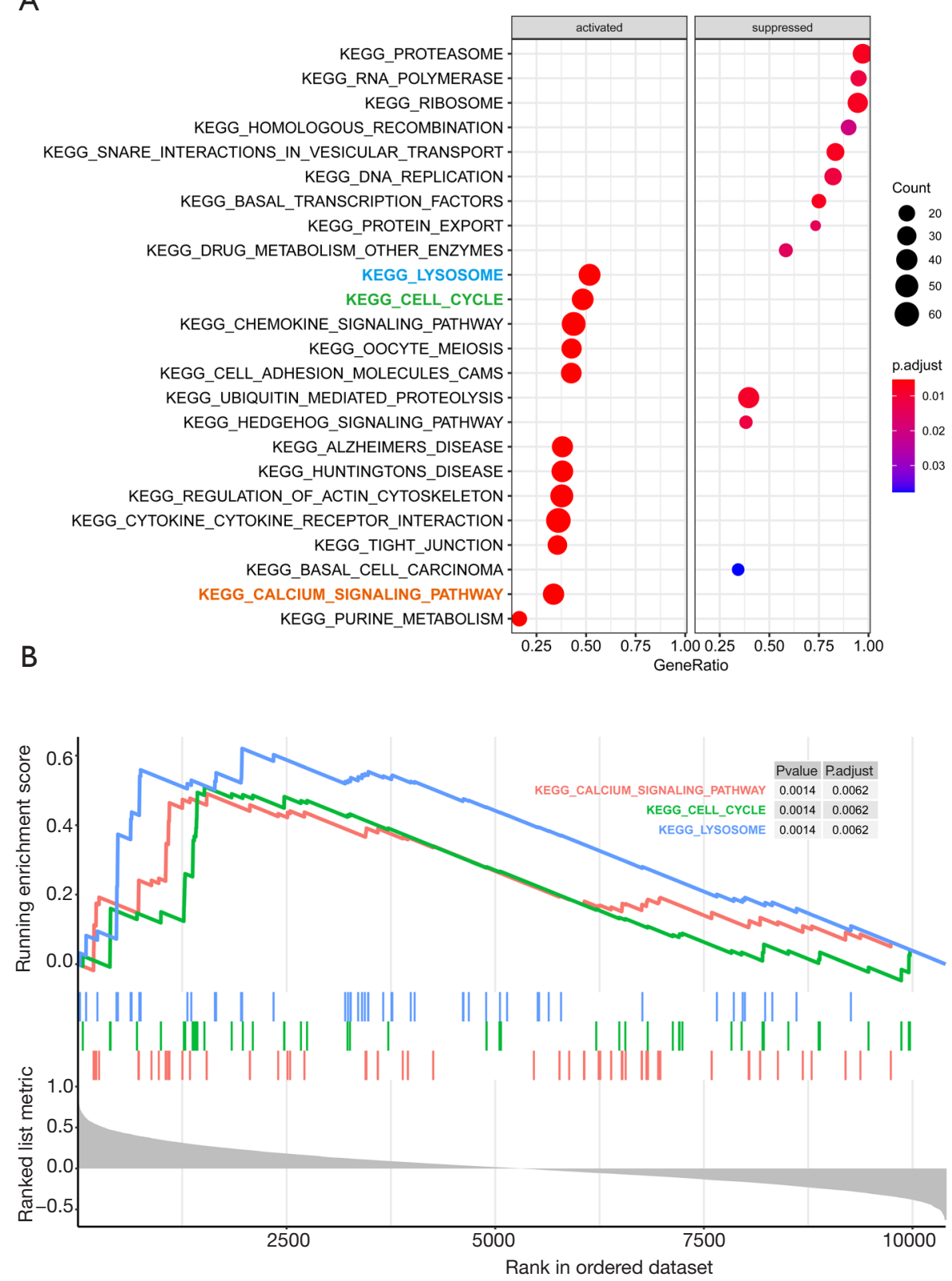

Figure 5 KEGG enrichment analysis of $I L 1 B$. (A) Upregulated and downregulated KEGG pathways related to changes in $I L 1 B$ expression levels in the process of receptivity recovery and repair of the endometrium. Pathways involved are shown in blue, green and red. (B) GSEA plot of lysosomal, cell cycle and calcium signalling pathways.

result to a high re-adhesion rate $(30-66 \%)$ of the uterine cavity and a low pregnancy rate (40\%) after surgery (3). Therefore, avoidance of postoperative adhesion reformation and restoration of fertility are of utmost importance after hysteroscopic adhesiolysis.

In our previous study, we reported a significant improvement in endometrium repair and prevention of adhesion reformation using freeze-dried amnion graft (1). In addition, Amer et al. (27) reported that amnion grafts effectively improve endometrial receptivity thereby promoting pregnancy, consistent with the pregnancy outcome of this study. The amnion graft regulates tissue repair by regulating the expression levels of cytokines $(28,29)$. Immune cells, especially macrophages, play a crucial 
A

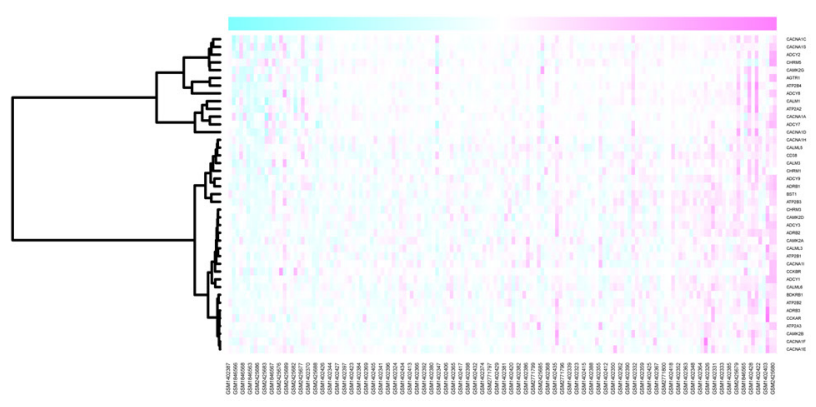

B

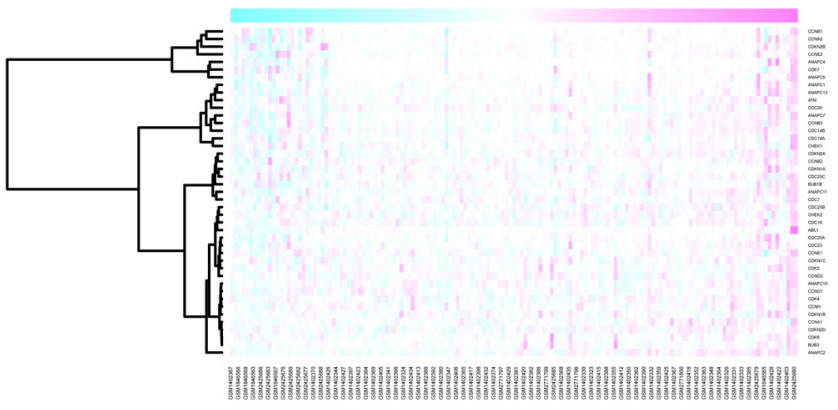

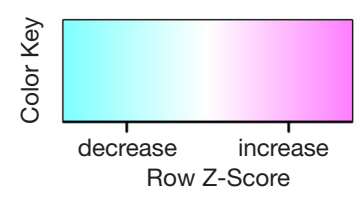

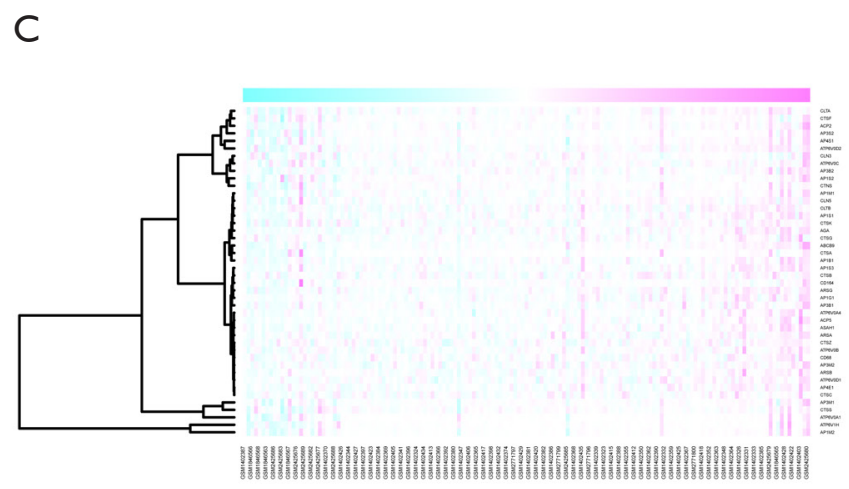

Figure 6 Heatmap showing KEGG pathways related to the functions of IL1B. (A) Heatmap of calcium signalling pathway. (B) Heatmap of cell cycle pathway. (C) Heatmap of lysosomal pathway. Red color represents upregulated genes while blue color represents downregulated genes.

role in the repair process after hysteroscopic adhesiolysis. Macrophage-related cytokines include IL1B, TNF- $\alpha$, and VEGF. The expression levels of these cytokines change to reflect the corresponding immune regulation process (7). However, a limited number of studies have evaluated the expression patterns of cytokines at different time points after hysteroscopic adhesiolysis. Therefore, we performed a randomized controlled trial on patients and measured cytokine concentrations in uterine exudates at different time points after hysteroscopic adhesiolysis. Cytokine expression patterns in the amnion group were found to be significantly different from those of the control group. In a previous study, Orhue et al. found that the repair phase of inflammatory responses occur in approximately seven days after hysteroscopic adhesiolysis (9). In our study, changing patterns in cytokine expression levels in the amnion group showed clear stratifications when compared to the control group. The $6^{\text {th }}$ day after surgery in the amnion group divided the inflammatory reaction stages of the endometrium into two phases. This stratification pattern was consistent to the findings reported by Orhue et al. (9). Immune pattern stratification may be dominated by macrophage changes. Xin et al. documented that immune regulation of macrophages promotes endometrial regeneration and fertility restoration. Immune responses after tissue trauma lasts for approximately 1-2 weeks. Immune response durations can significantly impact on the healing process. The first stage of immune response is dominated by M1 macrophages, which play a vital role in removing tissues, residual fibers, and tissue debris, as well as synthesizing pro-inflammatory cytokines and growth factors. In the late stage of inflammation, M2 macrophages and T-regs jointly promote repair of the endometrial tissues (7). Elevated expression levels of cytokines such as TNF- $\alpha$ in the early stages promote polarization of M1 macrophages to M2 macrophages, as well as the synthesis and secretion of $I L 1 B$ by macrophages. In addition, $I L 1 B$ in the early stages can act on $I L-1 R A$ to promote its synthesis and secretion. 
A
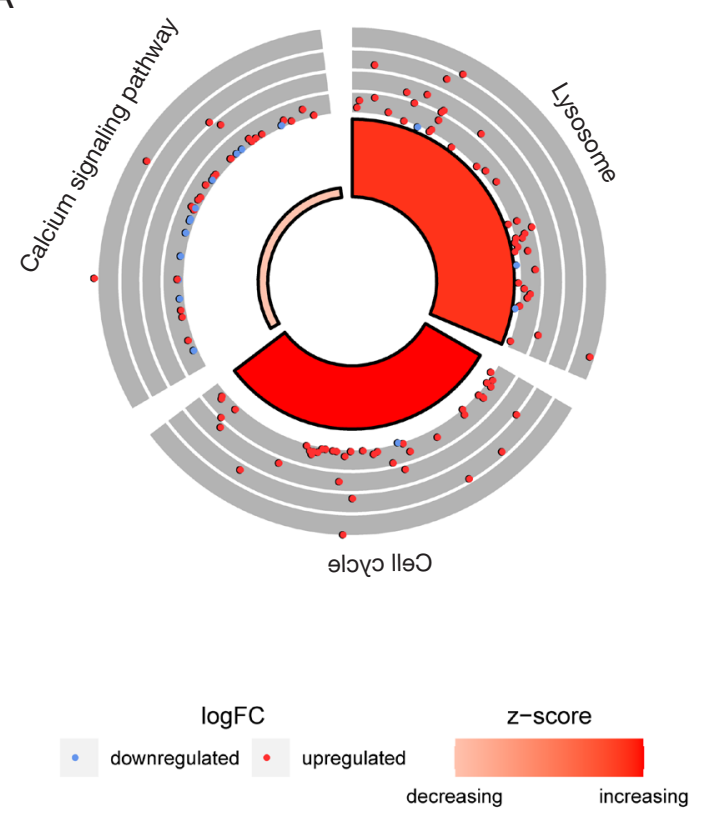

C
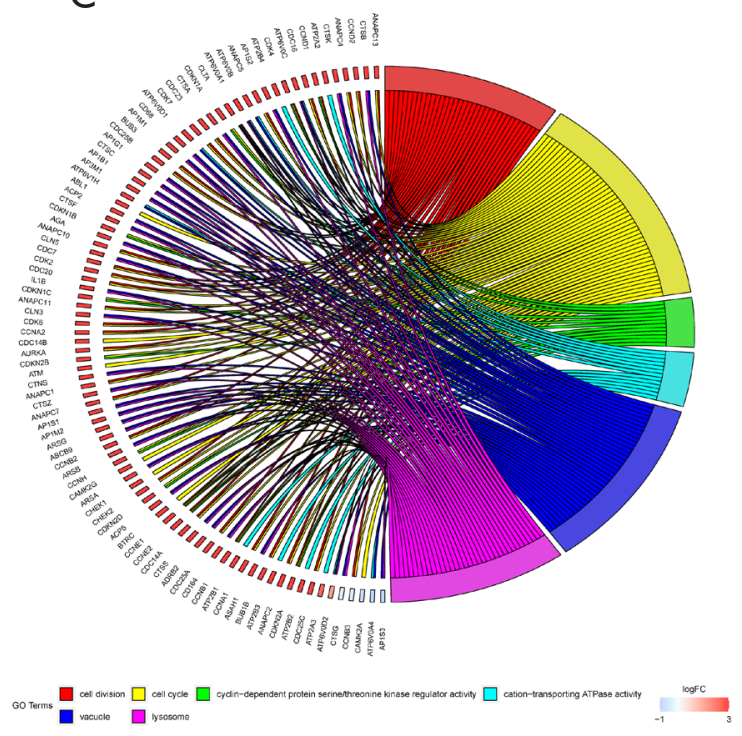

B

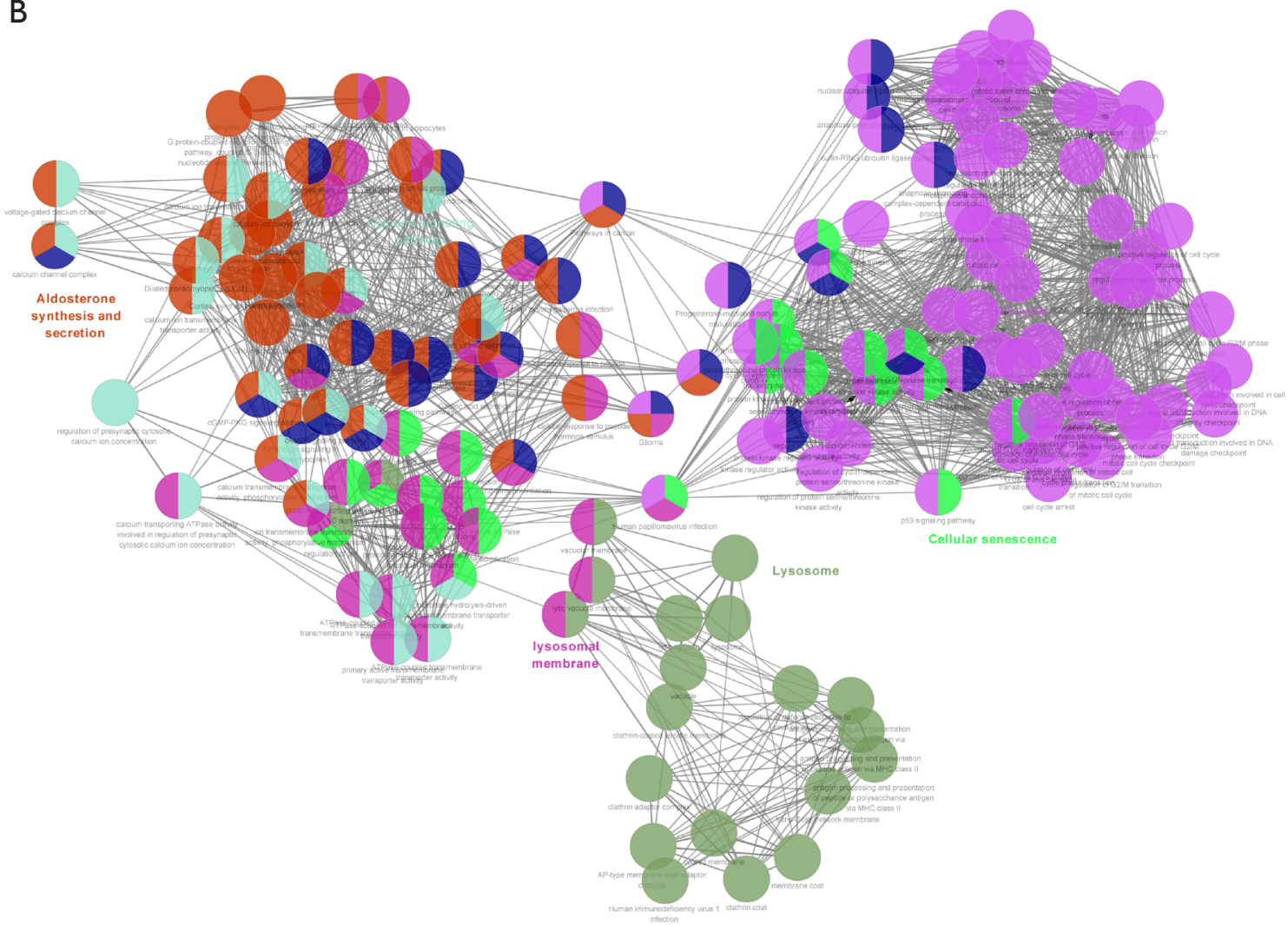

Figure 7 IL1B over-expression enrichment analysis. (A) GO Circle. From outside to inside: GO ID; upregulated and downregulated genes (red color represents upregulated genes, blue color represents downregulated genes); z-score. (B) KEGG and GO enrichment network diagram. Each color represents a KEGG pathway term. (C) GO Chord. The left side is the gene (level of fold change of the gene is colorcoded). The right side represents the different GO terms. Connected bands indicate that a gene is highly correlated to its corresponding GO terms. 
In this study, the peak of $I L 1 B$ secretion was delayed in the amnion group. This finding implies that the amnion graft prolongs the effect of repair phase by regulating cytokines. Furthermore, it suppresses the secretion of adhesion promoting factors during the repair period, which promotes endometrial tissue repair, reduces scar formation, and promotes endometrial receptivity recovery. Cluster analysis showed that the secretion patterns of $I L 1 B$ were not consistent with the levels of TNF- $\alpha$ and VEGF. From day 6 to day 7 after surgery, $I L 1 B$ secretion patterns in the amnion group were found to be altered. Besides, the aforementioned changes were accompanied by improved pregnancy outcomes. This observation implies that the repair phase of endometrial tissues is affected by amnion gifts, and it begins from the sixth to seventh day after hysteroscopic adhesiolysis. Changes in expression levels of $I L 1 B$ may play an important role in endometrial repair and receptivity.

IL-1 was first reported by Gray et al. in 1972 when culturing monocytes. The IL-1 family includes IL-1 $\alpha$, $I L 1 B$, IL-33, and the IL-1 receptor antagonist (IL-1RA) (30). Recently, the role of $I L 1 B$ in reproductive activities has been elucidated and is reported to play a key role in endometrial receptivity. Rossi et al. reported that $I L 1 B$ promotes endometrium proliferation and repair pathways before menstruation. In addition, $I L 1 B$ activates endometrial basal cells, thereby maintaining a healthy endometrial receptivity for women (6). In this study, microarray datasets of reduced endometrial receptivity and normal premenstrual endometrial tissues were retrieved from the GEO database for analysis. A history of uterine cavity procedures was used as the inclusion criteria to explore the effects and mechanisms of action of $I L 1 B$ on the endometrial repair process and recovery of endometrial receptivity.

In this clinical trial, we found that the expression levels of $I L 1 B$ mRNA in the normal group were significantly higher than for patients in the endometrial receptivity reduced group. This finding is consistent with those reported by Rossi et al. Enrichment analysis on $I L 1 B$-related genes was performed to evaluate the role of $I L 1 B$ in endometrial repair and receptivity. Enrichment analysis showed that genes that were positively correlated with $I L 1 B$ are mainly enriched in calcium signaling, cell cycle, and lysosome pathways. The specific mechanism is shown in Figure 8.

$I L 1 B$ is a potent pro-inflammatory cytokine that initiates and amplifies the effects associated with tissue damage responses. The production and release of $I L 1 B$ is a multistep process and is stimulated by damaged-associated molecular pattern molecules (DAMPs). IL1B is first synthesized into biologically inactive pro $I L 1 B$, which is processed by caspase-1 into mature, biologically active $I L 1 B$, and then released into the extracellular environment (31). Women with elevated endometrial receptivity have up-regulated IL1B expression levels in the endometrium (32). Besides, previous studies on endometriosis revealed that the expression levels of $I L 1 B$ in the abdominal cavity is high, implying that $I L 1 B$ is involved in the proliferation of endometrial cells (6). After culturing of human menstrual blood-derived stromal cells, Zhang et al. reported that cytokines such as $I L 1 B$ can stimulate cells to transform from the G1 phase to the $S$ phase (33). In this study, IL1B up-regulated CDKs protein through the NF-кB pathway, which positively regulates the cell cycle pathway and promotes endometrial basal cell transition from G1 phase to $\mathrm{S}$ phase. These activities further enhance the proliferation and crawling of endometrial cells covering the wound surface.

$I L 1 B$ plays a role in moderation of immune activation and positively regulates the lysosomal pathway through proinflammatory actions to promote phagocytosis. This activity destroys and ablates fibrin around the wound, prevents the fibrotic healing process of the endometrium, and inhibits adhesion formation after surgery. Moreover, $I L 1 B$ secretion depends on the exocytosis of secretory lysosomes (30). Findings from this study imply that $I L 1 B$ is involved in the lysosomal pathway, thereby affecting endometrial repair.

Notably, ILIB is involved in the calcium signaling pathway, where it regulates endometrial receptivity. In our previous study, we explored changes in calcium ion signaling pathway in the endometrium and junction zone (JZ). Abnormalities in this pathway can cause irregular contraction waves in the $\mathrm{JZ}$ of the uterus and inhibit endometrial receptivity (34). Besides, rhythmic contraction helps prevent the formation of fiber adhesions during the repair process. Tribe et al. (35) reported that $I L 1 B$ can cause changes in key calcium homeostasis mechanisms, thereby promoting the contraction of the myometrium. In addition, $I L 1 B$ enhances the expression of sarcoplasmic reticulum calcium ATPase (SERCA 2b) in human myometrial smooth muscle (HMSM) cells. Further, it promotes cyclopiazonic acid-induced calcium transients by activation of lanthanumsensitive, store-operated calcium entry.

Through randomized controlled designs, monitoring of cytokine expression levels and hierarchical clustering model analyses, combined with microarray analysis, this study explored the effects of changes in expression patterns of cytokines caused by freeze-dried amnion grafts in 


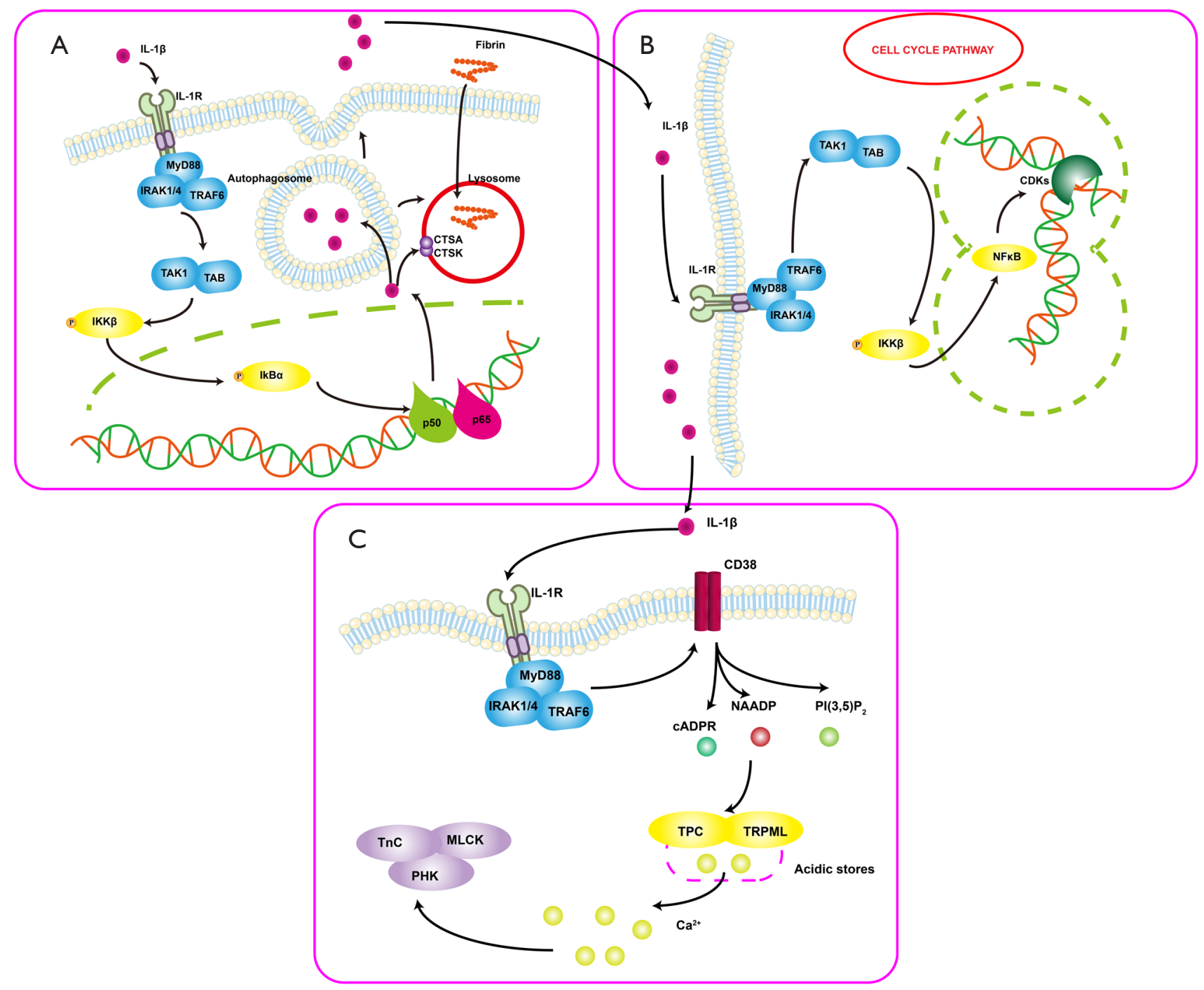

Figure 8 Different steps of interleukin (IL)-1 $\beta$ production pathway and signal transmission. (A) Priming, secretion and promotion of phagocytic functions of macrophages. IL-1 $\beta / \mathrm{IL}-1 \mathrm{R}$ activates IRAK1/4 and TRAF6 promotes its production. Secretion of IL-1 $\beta$ is regulated by a secretory lysosome, and IL- $1 \beta$ further upregulates the lysosomal pathway. (B) IL-1 $\beta$ promotes endometrial cell proliferation and activates CDKs through the NF- $\mathrm{KB}$ pathway to promote transformation of cells from $\mathrm{G} 1$ to $\mathrm{S}$ phase. (C) IL- $1 \beta$ promotes $\mathrm{CD} 38$ and its downstream pathways \{cADPR, NAADP and PI [3,5] P2 $\}$ by binding IL-1R. NAADP activates a distinct $\mathrm{Ca}^{2+}$ release mechanism on separate acidic $\mathrm{Ca}^{2+}$ stores. $\mathrm{Ca}^{2+}$ release through the NAADP-sensitive mechanism may also have a feedback mechanism onto RYRs or IP3Rs. This pathway may be activated in the uterus junction zone (JZ) and preserve its regular contraction waves.

endometrial receptivity after hysteroscopic adhesiolysis. However, this study had some limitations. Patients involved in this study showed moderate to severe IUA, therefore, for ethical reasons we could not collect the endometrium of patients after hysteroscopic adhesiolysis for further analysis. Therefore, we retrieved endometrium microarrays from the GEO database for analysis. We used the history of uterine cavity procedure as the inclusion criteria to evaluate the effect of $I L 1 B$ on the recovery of endometrial receptivity during endometrial repair. Secondly, we did not verify the changes in cytokine expression levels observed in patients after hysteroscopic adhesiolysis. To ensure accuracy of the results, we performed DEGs analysis and the results were consistent with those of GSEA. In addition, the findings 
of this study are consistent with findings from previous studies. Therefore, we conclude that $I L 1 B$ is a "sign" of the recovery of endometrial receptivity after hysteroscopic adhesiolysis. However, further studies using in vitro cell cultures and animal models should be performed to explore the effects of $I L 1 B$ and its related pathways on the recovery of endometrial receptivity. Thus, it cannot be concluded that $I L 1 B$ is the deciding parameter. Finally, all data in this study were normalized and data deviations generated by different batches and different machines were corrected through the Combat calculation method, which directly removes known batch effects through the SVA package. To prevent the bias generated by different platforms, we used a method by Leek et al. (36) and performed prior distribution assumptions and PCA. The results were consistent with those from the previous study, and no significant batch effects were found. However, details of the original data, including race, processing method, and different batches may produce inevitable effects, therefore, the results should be verified further through experiments.

\section{Conclusions}

In summary, this study established a postoperative cytokine change model and used the GEO database to evaluate cytokine expression patterns in patients who had undergone hysteroscopic adhesiolysis. The findings showed that freeze-dried amnion graft promotes endometrial repair and receptivity recovery by changing the expression patterns of cytokines. Therefore, we concluded that changes in expression patterns of cytokines after surgery promote endometrial repair and prevent re-adhesion of the uterine cavity. Furthermore, changes in cytokine expression patterns in the uterine exudate can be used as an indicator for postoperative evaluation and outcome prediction.

\section{Acknowledgments}

The paper is edited by Home for researchers' editorial team.

Funding: This study was financially supported by the National Key Research and Development Program of China (2018YFC1004803).

\section{Footnotes}

Reporting Checklist: The authors have completed the MDAR reporting checklist (available at http://dx.doi.org/10.21037/ atm-21-195).

Data Sharing Statement: Available at http://dx.doi. org/10.21037/atm-21-195

Peer Review File: Available at http://dx.doi.org/10.21037/ atm-21-195

Conflicts of Interest: All authors have completed the ICMJE uniform disclosure form (available at http://dx.doi. org/10.21037/atm-21-195). The authors have no conflicts of interest to declare.

Ethical Statement: The authors are accountable for all aspects of the work in ensuring that questions related to the accuracy or integrity of any part of the work are appropriately investigated and resolved. Ethical approval for this study was obtained from the Research Ethics Committee of the Beijing Obstetrics and Gynecology Hospital. Experiments were performed (under protocol number 2017-KY-082-02) according to the Helsinki Declaration of 1975 (revised in 2013). Patients eligible for surgery were required to sign an informed consent the night before surgery.

Open Access Statement: This is an Open Access article distributed in accordance with the Creative Commons Attribution-NonCommercial-NoDerivs 4.0 International License (CC BY-NC-ND 4.0), which permits the noncommercial replication and distribution of the article with the strict proviso that no changes or edits are made and the original work is properly cited (including links to both the formal publication through the relevant DOI and the license). See: https://creativecommons.org/licenses/by-nc-nd/4.0/.

\section{References}

1. Gan L, Duan H, Sun FQ, et al. Efficacy of freeze-dried amnion graft following hysteroscopic adhesiolysis of severe intrauterine adhesions. Int J Gynaecol Obstet 2017;137:116-22.

2. Zheng F, Zhu B, Liu Y, et al. Meta-analysis of the use of amniotic membrane to prevent recurrence of intrauterine adhesion after hysteroscopic adhesiolysis. Int J Gynaecol Obstet 2018;143:145-9.

3. AAGL Practice Report: Practice Guidelines on Intrauterine Adhesions Developed in Collaboration With the European Society of Gynaecological Endoscopy 
(ESGE). J Minim Invasive Gynecol 2017;24:695-705.

4. Johary J, Xue M, Zhu X, et al. Efficacy of estrogen therapy in patients with intrauterine adhesions: systematic review. J Minim Invasive Gynecol 2014;21:44-54.

5. Abudukeyoumu A, Li MQ, Xie F. Transforming growth factor- $\beta 1$ in intrauterine adhesion. Am J Reprod Immunol 2020;84:e13262.

6. Rossi M, Sharkey AM, Viganò P, et al. Identification of genes regulated by interleukin-1beta in human endometrial stromal cells. Reproduction 2005;130:721-9.

7. Xin L, Lin X, Zhou F, et al. A scaffold laden with mesenchymal stem cell-derived exosomes for promoting endometrium regeneration and fertility restoration through macrophage immunomodulation. Acta Biomater 2020;113:252-66.

8. Lee YS, Wysocki A, Warburton D, et al. Wound healing in development. Birth Defects Res C Embryo Today 2012;96:213-22.

9. Orhue AAE, Aziken ME, Igbefoh JO. A comparison of two adjunctive treatments for intrauterine adhesions following lysis. Int J Gynaecol Obstet 2003;82:49-56.

10. Gan L, Duan H, Xu Q, et al. Human amniotic mesenchymal stromal cell transplantation improves endometrial regeneration in rodent models of intrauterine adhesions. Cytotherapy 2017;19:603-16.

11. Zhang Z, Murtagh F, Van Poucke S, et al. Hierarchical cluster analysis in clinical research with heterogeneous study population: highlighting its visualization with $\mathrm{R}$. Ann Transl Med 2017;5:75.

12. Petushkova NA, Pyatnitskiy MA, Rudenko VA, et al. Applying of hierarchical clustering to analysis of protein patterns in the human cancer-associated liver. PLoS One 2014;9:e103950.

13. Moss TJ, Lake DE, Calland JF, et al. Signatures of Subacute Potentially Catastrophic Illness in the ICU: Model Development and Validation. Crit Care Med 2016;44:1639-48.

14. O'Leary JD, Wunsch H, Leo AM, et al. Hospital admission on weekends for patients who have surgery and 30-day mortality in Ontario, Canada: A matched cohort study. PLoS Med 2019;16:e1002731.

15. Koot YE, van Hooff SR, Boomsma CM, et al. An endometrial gene expression signature accurately predicts recurrent implantation failure after IVF. Sci Rep 2016;6:19411.

16. Pathare ADS, Zaveri K, Hinduja I. Downregulation of genes related to immune and inflammatory response in IVF implantation failure cases under controlled ovarian stimulation. Am J Reprod Immunol 2017. doi: 10.1111/ aji.12679.

17. Gautier L, Cope L, Bolstad BM, et al. affy--analysis of Affymetrix GeneChip data at the probe level. Bioinformatics 2004;20:307-15.

18. Li Y, Bai W, Zhang L. The Overexpression of CD80 and ISG15 Are Associated with the Progression and Metastasis of Breast Cancer by a Meta-Analysis Integrating Three Microarray Datasets. Pathol Oncol Res 2020;26:443-52.

19. Yoneya T, Miyazawa T. Integration of pre-normalized microarray data using quantile correction. Bioinformation 2011;5:382-5.

20. Shakya K, Ruskin HJ, Kerr G, et al. Comparison of microarray preprocessing methods. Adv Exp Med Biol 2010;680:139-47.

21. NCBI Resource Coordinators. Database resources of the National Center for Biotechnology Information. Nucleic Acids Res 2018;46:D8-13.

22. NCBI Resource Coordinators. Database resources of the National Center for Biotechnology Information. Nucleic Acids Res 2015;43:D6-17.

23. Leek JT. svaseq: removing batch effects and other unwanted noise from sequencing data. Nucleic Acids Res 2014;42:e161.

24. Yu G, Wang LG, Han Y, et al. clusterProfiler: an R package for comparing biological themes among gene clusters. Omics 2012;16:284-7.

25. Ritchie ME, Phipson B, Wu D, et al. limma powers differential expression analyses for RNA-sequencing and microarray studies. Nucleic Acids Res 2015;43:e47.

26. Konci R, Caminsky N, Tulandi T, et al. Supplements to Conventional Treatment After Hysteroscopic Lysis of Intrauterine Adhesions: A Systematic Review. J Obstet Gynaecol Can 2020;42:984-1000.

27. Amer MI, Abd-El-Maeboud KHI, Abdelfatah I, et al. Human amnion as a temporary biologic barrier after hysteroscopic lysis of severe intrauterine adhesions: pilot study. J Minim Invasive Gynecol 2010;17:605-11.

28. Peng X, Li T, Zhao Y, et al. Safety and Efficacy of Amnion Graft in Preventing Reformation of Intrauterine Adhesions. J Minim Invasive Gynecol 2017;24:1204-10.

29. Jirsova K, Jones GLA. Amniotic membrane in ophthalmology: properties, preparation, storage and indications for grafting-a review. Cell Tissue Bank 2017;18:193-204.

30. Claude-Taupin A, Bissa B, Jia J, et al. Role of autophagy in IL-1 $\beta$ export and release from cells. Semin Cell Dev Biol 2018;83:36-41. 
31. Rébé C, Ghiringhelli F. Interleukin- $1 \beta$ and Cancer. Cancers 2020;12:1791.

32. Wang H, Shi G, Li M, et al. Correlation of IL-1 and HB-EGF with endometrial receptivity. Exp Ther Med 2018;16:5130-6.

33. Zhang S, Li P, Yuan Z, et al. Effects of platelet-rich plasma on the activity of human menstrual blood-derived stromal cells in vitro. Stem Cell Res Ther 2018;9:48.

34. Wang S, Duan H, Li B. Rapid Effects of Oestrogen on Intracellular $\mathrm{Ca}(2+)$ in the Uterine Junctional Myometrium of

Cite this article as: Li B, Duan H, Wang S, Wang Y, Chang Y, Guo Z, Li Y. Hierarchical cluster analysis in the study of the effect of cytokine expression patterns on endometrial repair and receptivity after hysteroscopic adhesiolysis. Ann Transl Med 2021;9(9):746. doi: 10.21037/atm-21-195
Patients With and Without Adenomyosis in Different Phases of the Menstrual Cycle. Reprod Sci 2020;27:1992-2001.

35. Tribe RM, Moriarty P, Dalrymple A, et al. Interleukin1 beta induces calcium transients and enhances basal and store operated calcium entry in human myometrial smooth muscle. Biol Reprod 2003;68:1842-9.

36. Leek JT, Scharpf RB, Bravo HC, et al. Tackling the widespread and critical impact of batch effects in highthroughput data. Nat Rev Genet 2010;11:733-9. 
Table S1 Comparison of the TNF- $\alpha$ concentration between the two groups at different postoperative time points $(\bar{x} \pm s, \mathrm{n}=15)$

\begin{tabular}{|c|c|c|c|c|c|c|c|c|c|c|}
\hline \multirow{2}{*}{ Group } & \multicolumn{4}{|c|}{ Postoperative time points } & \multicolumn{4}{|c|}{ Postoperative time points } & \multirow{2}{*}{$F, P$} & \multirow{2}{*}{$\mathrm{Q}, \mathrm{P}$} \\
\hline & $3 \mathrm{~h}$ & $24 \mathrm{~h}$ & $48 \mathrm{~h}$ & $72 \mathrm{~h}$ & $4 d$ & $5 d$ & $6 d$ & $7 d$ & & \\
\hline Study group & $212.91 \pm 38.08$ & $276.13 \pm 30.01$ & $279.35 \pm 29.52$ & $275.08 \pm 31.17$ & $271.67 \pm 28.62$ & $259.77 \pm 38.25$ & $250.69 \pm 28.83$ & $243.56 \pm 43.99$ & $7.241,0.000$ & $Q_{11-6}=3.990,<0.05$ \\
\hline Control group & $247.83 \pm 48.71$ & $299.87 \pm 31.13$ & $309.18 \pm 35.20$ & $305.47 \pm 37.62$ & $300.78 \pm 31.14$ & $296.39 \pm 41.13$ & $289.65 \pm 31.73$ & $285.06 \pm 38.20$ & $3.909,0.000$ & $Q_{11-6}=2.485,>0.05$ \\
\hline $\mathrm{t}$ & -2.187 & -2.126 & -2.515 & -2.409 & -2.666 & -2.525 & -3.520 & -2.759 & & \\
\hline$P$ & 0.037 & 0.042 & 0.018 & 0.023 & 0.013 & 0.018 & 0.002 & 0.010 & & \\
\hline
\end{tabular}

The SNK-q test was performed for comparisons within the same group at different time points.

Table S2 Comparison of the VEGF concentration between the two groups at different postoperative time points $(\bar{x} \pm s, n=15)$

\begin{tabular}{|c|c|c|c|c|c|c|c|c|c|c|}
\hline \multirow{2}{*}{ Group } & \multicolumn{4}{|c|}{ Postoperative time points } & \multicolumn{4}{|c|}{ Postoperative time points } & \multirow{2}{*}{$F, P$} & \multirow{2}{*}{$\mathrm{Q}, \mathrm{P}$} \\
\hline & $3 \mathrm{~h}$ & $24 \mathrm{~h}$ & $48 \mathrm{~h}$ & $72 \mathrm{~h}$ & $4 d$ & $5 d$ & $6 d$ & $7 d$ & & \\
\hline Study group & $380.11 \pm 113.25$ & $529.69 \pm 124.91$ & $601.15 \pm 97.98$ & $629.24 \pm 101.38$ & $605.74 \pm 121.63$ & $558.31 \pm 113.27$ & $477.87 \pm 117.91$ & $428.16 \pm 104.35$ & $9.265,0.000$ & $Q_{11-7}=6.974,<0.05$ \\
\hline Control group & $474.87 \pm 108.37$ & $713.47 \pm 110.20$ & $708.42 \pm 129.15$ & $688.37 \pm 105.37$ & $689.98 \pm 110.03$ & $655.28 \pm 97.63$ & $601.28 \pm 100.39$ & $564.59 \pm 119.92$ & $7.042,0.000$ & $Q_{11-5}=5.169,<0.05$ \\
\hline $\mathrm{t}$ & -2.341 & -4.273 & -2.563 & -1.566 & -1.989 & -2.512 & -3.087 & -3.324 & & \\
\hline $\mathrm{P}$ & 0.027 & 0.000 & 0.016 & 0.129 & 0.057 & 0.018 & 0.005 & 0.003 & & \\
\hline
\end{tabular}

The SNK-q test was performed for comparisons within the same group at different time points.

Table S3 Comparison of the IL-1 concentration between the two groups at different postoperative time points $(\bar{x} \pm s, \mathrm{n}=15)$

\begin{tabular}{|c|c|c|c|c|c|c|c|c|c|c|}
\hline \multirow{2}{*}{ Group } & \multicolumn{4}{|c|}{ Postoperative time points } & \multicolumn{4}{|c|}{ Postoperative time points } & \multirow{2}{*}{$F, P$} & \multirow{2}{*}{$Q, P$} \\
\hline & $3 \mathrm{~h}$ & $24 \mathrm{~h}$ & $48 \mathrm{~h}$ & $72 \mathrm{~h}$ & $4 d$ & $5 d$ & $6 d$ & $7 d$ & & \\
\hline Study group & $53.81 \pm 20.32$ & $71.66 \pm 14.72$ & $70.63 \pm 13.22$ & $71.01 \pm 15.35$ & $77.85 \pm 12.11$ & $81.34 \pm 14.39$ & $84.41 \pm 16.31$ & $80.62 \pm 14.77$ & $6.455,0.000$ & $Q_{11-10}=0.923,>0.05$ \\
\hline Control group & $75.12 \pm 13.45$ & $83.97 \pm 14.73$ & $87.73 \pm 9.98$ & $95.34 \pm 19.77$ & $95.23 \pm 14.36$ & $101.35 \pm 19.53$ & $97.03 \pm 17.01$ & $89.89 \pm 13.83$ & $4.105,0.000$ & $Q_{11-9}=2.736,>0.05$ \\
\hline $\mathrm{t}$ & -3.387 & -2.289 & -3.998 & -3.765 & -3.583 & -3.195 & -2.074 & -1.774 & & \\
\hline$P$ & 0.002 & 0.030 & 0.000 & 0.001 & 0.001 & 0.004 & 0.047 & 0.087 & & \\
\hline
\end{tabular}

The SNK-q test was performed for comparisons within the same group at different time points. 


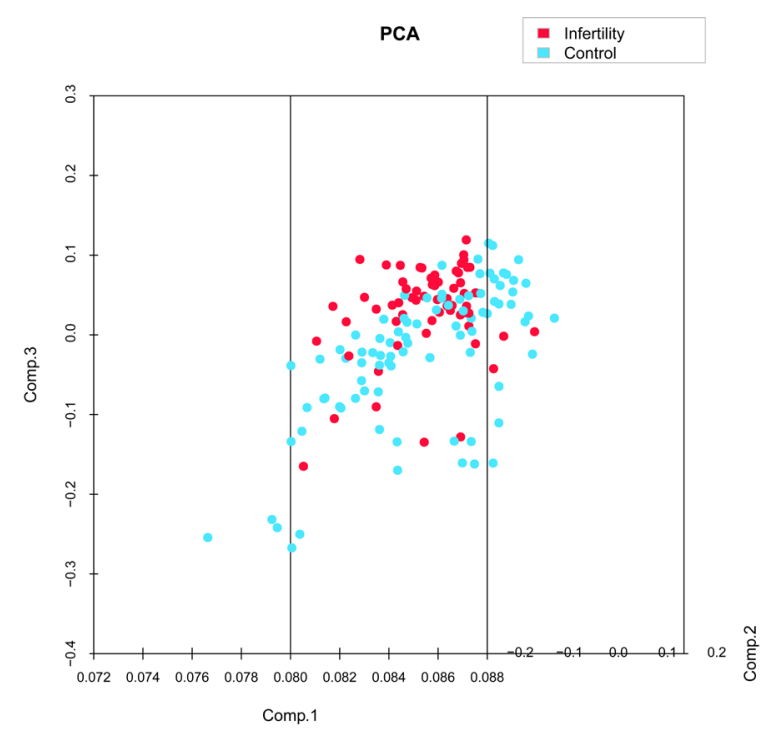

Figure S1 PCA plot of the data for each group. 